\title{
Detection Pork Oil and Palm Oil Using Light Intensity Parameters
}

\author{
Virginia Fahriza Amaliya*, Frida Agung Rakhmadi \\ Physics Departement, Facullty of Science and Technologi, UIN Sunan Kalijaga \\ Jl. Marsda Adisucipto No.1 Yogyakarta 55281, Indonesia. Tel. +6285708667769. \\ Email*: virginiafahrizaa@gmail.com
}

\begin{abstract}
To distinguish between pork oil and palm oil, we can use the characteristics of light absorbtion from the material. So, in this research we set a detection method using light intensity as the parameters. Equipment that we used are LDR sensors and red LED as the light source. Pork oil and palm oil were varied into two types of treatment, namely oil with no filtering and a single filtering. In this experiment we got that pork oil has higher light intensity than palm oil. It means that palm oil more absorb the light than pork oil. So, we conclude that light intensity can distinguish the type of oil. Further study is needed so we can develope more accurate and simple detection tool to determine the type of oil especially pork oil in order to give information about halal food for muslim.
\end{abstract}

Keywords: LED, LDR, light intensity, palm oil, pork oil

Abbreviations: LDR (Light Dependent Resistor), LED (Light Emitting Diode)

\section{INTRODUCTION}

The development of food products must be scrutinized with safety. Food safety is very important to prevent food from health hazards and does not conflict with the religion, beliefs, culture of the community so that it is safe for consumption. Processed food products can be made from animal or vegetable.

Cooking oil is one of the processed products from animals or vegetables that is needed by the community in order to meet their daily needs. Vegetable cooking oil is widely used by the community such as palm oil. Palm oil comes from oil palm plants which are obtained by extracting the fruit of the plant. Palm oil is one of the most widely produced and consumed oil by the people. Low prices and oxidative stability, when used for frying are optional. Palm oil, including oil that has high levels of saturated fat. Palm oil is semi-solid at room temperature and has saturated fat.

But not a few people who use animal oil because the taste and texture are better than vegetable oil. One of the animal oils that are widely circulating in the market and is often used in various restaurants, namely pork oil. Pork oil has 60 percent unsaturated fat content compared to palm oil which has 45 percent unsaturated fat.

Processed pork itself in Islam, including illicit food. So it must be avoided by Muslims. In addition, foods that have been mixed with illicit food, we must also avoid. Said the Prophet Muhammad, "The halal is clear, and even the haram is clear: and between them, there are mushyabihat (vague, unclear halal unclear) most people do not know the law. Whoever is careful of the case of doubtfulness, truly he has saved religion and his pride. "(H.R. Muslim). So that we can avoid consuming foods that are mixed with unclean foods, we must know the characteristics of these foods.

This study will examine the characteristics of palm oil with pork oil. The characteristics examined include physical characteristics namely the light intensity parameters. The characteristics shown will produce information on the difference between palm oil and pork oil. So that it is expected to provide information about food security and food safety in general and sharia.

\section{MATERIALS AND METHODS}

\section{Study area}

Light Diode Resistor (LDR) is a resistor type sensor where the working principle is based on the resistance value. The resistance depends on the intensity of the light received. The resistance value on the LDR will decrease if the received light is bright and the resistance value will increase if it is in a dark condition. It can be said that the LDR will conduct an electric current if it receives a certain amount of light intensity (bright conditions) and will inhibit the electric current if it does not receive a certain amount of light intensity (dark conditions).

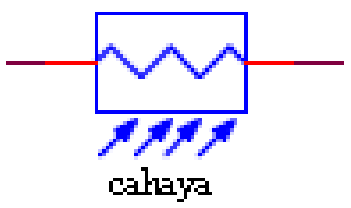

Figure 1. Light Diode Resistor (LDR). 
This LDR sensor can be seen in Figure 1, where the LDR sensor which is exposed to the sensor light will conduct current. The output of the sensor is an analog voltage.

Data from the sensor will be controlled, managed and processed using the Arduino Uno microcontroller. Arduino has a basic setup and loop functions. The setup function is a function used for initialization and will only be executed once when the program is run. While the loop function itself is a function that is used as the core of the program created, and will be run repeatedly as long as the program logic conditions are met and the power is not interrupted in Arduino.

\section{Procedures}

The design of the tool is made by designing the detection device that the system will make. This stage consists of the stages of making hardware and software designs.

\section{Hardware design}

The design to be made according to the circuit schematic in Figure 2

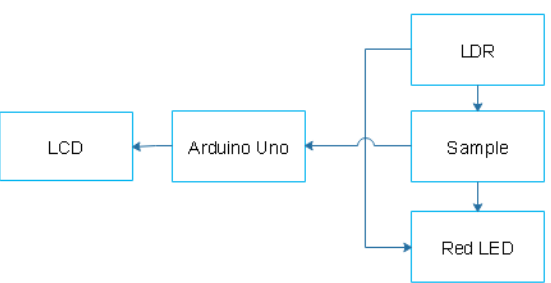

Figure 2. Circuit schematic

The design of this detection device is made using a voltage divider (LED and LDR) sequence which is used as input in the dark box as a sample test place where the light at zero light source intensity shows a constant value. The results of the sensor readings in analog voltage will be converted into digital form by the microcontroller and displayed to the LCD $16 \times 2$. This data will also be displayed and stored on the laptop during the test. The overall schematic of the system can be seen in Figure 3.

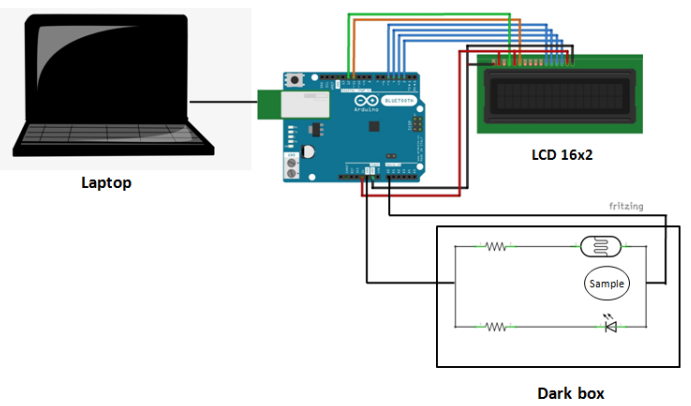

Figure 3. The overall schematic of the system

\section{Software design}

The design of the software contains commands that are used to run the Arduino Uno microcontroller so that the output of the desired sample differentiator can be known.
Sketch program on this system created using Arduino IDE 1.81. The sensor reading program follows the flow diagram shown in Figure 4.

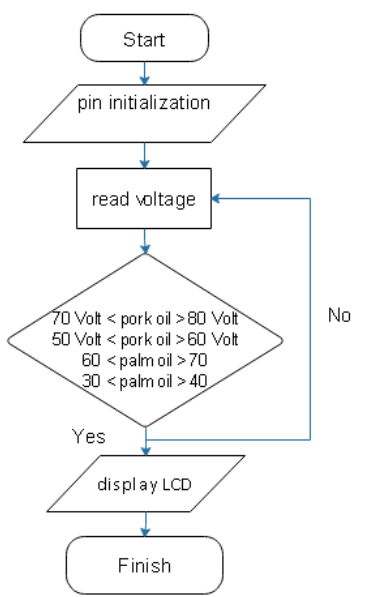

Figure 4. Flow diagram program

\section{Data analysis}

Data analysis aims to compare the data characteristics of palm oil and bulk and filtered pork oil with twice filters. Data analysis was performed using analysis of sample testing to determine differences in the characteristics of the samples used. Sample testing with light intensity parameters performed in a dark place (constant light).

Tests that produce data such that the percentage of accuracy of the detection system can be determined based on data processing.

$$
\text { Percentage of Accuracy }=\frac{\text { (n test }- \text { number of tests that failed }}{\mathrm{n} \text { test }} \times 100 \%
$$

Information:

$\mathrm{n}$ detection $=$ number of testing tools

\section{RESULTS AND DISCUSSION}

This characteristic test is carried out in a dark box as a place to test samples where the light at zero light source intensity shows a constant value. The manipulation variables are bulk palm oil, filtering twice the oil, pork oil without filtering and pork oil twice filtering.

In the cooking oil detection system that has been made, it has a working principle which is a voltage divider circuit where there are 2 main parts namely the light source that comes from the Laser and the light sensor that uses LDR, between them the test samples to be detected. So that the beam of light that comes from the Laser will be transmitted by the sample after that beam of light that passes through the sample will be detected by an LDR sensor that has been controlled in the Arduino microcontroller program, the input is the intensity of the light and the resulting output is a voltage.

To be able to make the distinguishing parameters of the sample to be tested, that is used data in the form of 
a voltage limit value of the characteristics of the two samples (according to the Arduino software program). Distinguishing parameters of the output after scanning by the results of the LDR sensor control, which is an indicator on the LCD where if the sample is detected palm oil, then the LCD will display the cooking oil inscription, as well as if the sample is detected pork oil then the LCD will display the writing pork oil.

Table 1. Result of the Manipulation Variables.

\begin{tabular}{|c|c|c|c|c|c|}
\hline \multirow{2}{*}{$\begin{array}{l}\text { Sample } \\
\text { test }\end{array}$} & \multicolumn{4}{|c|}{ Indicator } & \multirow[b]{2}{*}{$\operatorname{Exp}$} \\
\hline & Bulk Palm Oil & $\begin{array}{c}\text { Palm Oil Twice } \\
\text { Filtered }\end{array}$ & $\begin{array}{c}\text { Pork Oil Without } \\
\text { Filtering }\end{array}$ & $\begin{array}{l}\text { Pork Oil Twice } \\
\text { filtering }\end{array}$ & \\
\hline 1 & $\checkmark$ & $\checkmark$ & $\checkmark$ & $\checkmark$ & success \\
\hline 2 & $\checkmark$ & $\checkmark$ & $\checkmark$ & $\checkmark$ & success \\
\hline 3 & $\checkmark$ & $\checkmark$ & $\checkmark$ & $\checkmark$ & success \\
\hline 4 & $\checkmark$ & $\checkmark$ & $\checkmark$ & $\checkmark$ & success \\
\hline 5 & $\checkmark$ & $\checkmark$ & $\checkmark$ & $\checkmark$ & success \\
\hline 6 & $\checkmark$ & $\checkmark$ & $\checkmark$ & $\checkmark$ & success \\
\hline 7 & $\checkmark$ & $\checkmark$ & $\checkmark$ & $\checkmark$ & success \\
\hline 8 & $\checkmark$ & $\checkmark$ & $\checkmark$ & $\checkmark$ & success \\
\hline 9 & $\checkmark$ & $\checkmark$ & $\checkmark$ & $\checkmark$ & success \\
\hline 10 & $\checkmark$ & $\checkmark$ & $\checkmark$ & $\checkmark$ & success \\
\hline 11 & $\checkmark$ & $\checkmark$ & $\checkmark$ & $\checkmark$ & success \\
\hline 12 & $\checkmark$ & $\checkmark$ & $\checkmark$ & $\checkmark$ & success \\
\hline 13 & $\checkmark$ & $\checkmark$ & $\checkmark$ & $\checkmark$ & success \\
\hline 14 & $\checkmark$ & $\checkmark$ & $\checkmark$ & $\checkmark$ & success \\
\hline 15 & $\checkmark$ & $\checkmark$ & $\checkmark$ & $\checkmark$ & success \\
\hline 16 & $\checkmark$ & $\checkmark$ & $\checkmark$ & $\checkmark$ & success \\
\hline 17 & $\checkmark$ & $\checkmark$ & $\checkmark$ & $\checkmark$ & success \\
\hline 18 & $\checkmark$ & $\checkmark$ & $\checkmark$ & $\checkmark$ & success \\
\hline 19 & $\checkmark$ & $\checkmark$ & $\checkmark$ & $\checkmark$ & success \\
\hline 20 & $\checkmark$ & $\checkmark$ & $\checkmark$ & $\checkmark$ & success \\
\hline 21 & $\checkmark$ & $\checkmark$ & $\checkmark$ & $\checkmark$ & success \\
\hline 22 & $\checkmark$ & $\checkmark$ & $\checkmark$ & $\checkmark$ & success \\
\hline 23 & $\checkmark$ & $\checkmark$ & $\checkmark$ & $\checkmark$ & success \\
\hline 24 & $\checkmark$ & $\checkmark$ & $\checkmark$ & $\checkmark$ & success \\
\hline 25 & $\checkmark$ & $\checkmark$ & $\checkmark$ & $\checkmark$ & success \\
\hline 26 & $\checkmark$ & $\checkmark$ & $\checkmark$ & $\checkmark$ & success \\
\hline 27 & $\checkmark$ & $\checkmark$ & $\checkmark$ & $\checkmark$ & success \\
\hline 28 & $\checkmark$ & $\checkmark$ & $\checkmark$ & $\checkmark$ & success \\
\hline 29 & $\checkmark$ & $\checkmark$ & $\checkmark$ & $\checkmark$ & success \\
\hline 30 & $\checkmark$ & $\checkmark$ & $\checkmark$ & $\checkmark$ & success \\
\hline 31 & $\checkmark$ & $\checkmark$ & $\checkmark$ & $\checkmark$ & success \\
\hline 32 & $\checkmark$ & $\checkmark$ & $\checkmark$ & $\checkmark$ & success \\
\hline 33 & $\checkmark$ & $\checkmark$ & $\checkmark$ & $\checkmark$ & success \\
\hline 34 & $\checkmark$ & $\checkmark$ & $\checkmark$ & $\checkmark$ & success \\
\hline 35 & $\checkmark$ & $\checkmark$ & $\checkmark$ & $\checkmark$ & success \\
\hline 36 & $\checkmark$ & $\checkmark$ & $\checkmark$ & $\checkmark$ & success \\
\hline 37 & $\checkmark$ & $\checkmark$ & $\checkmark$ & $\checkmark$ & success \\
\hline 38 & $\checkmark$ & $\checkmark$ & $\checkmark$ & $\checkmark$ & success \\
\hline 39 & $\checkmark$ & $\checkmark$ & $\checkmark$ & $\checkmark$ & success \\
\hline 40 & $\checkmark$ & $\checkmark$ & $\checkmark$ & $\checkmark$ & success \\
\hline 41 & $\checkmark$ & $\checkmark$ & $\checkmark$ & $\checkmark$ & success \\
\hline 42 & $\checkmark$ & $\checkmark$ & $\checkmark$ & $\checkmark$ & success \\
\hline 43 & $\checkmark$ & $\checkmark$ & $\checkmark$ & $\checkmark$ & success \\
\hline 44 & $\checkmark$ & $\checkmark$ & $\checkmark$ & $\checkmark$ & success \\
\hline 45 & $\checkmark$ & $\checkmark$ & $\checkmark$ & $\checkmark$ & success \\
\hline 46 & $\checkmark$ & $\checkmark$ & $\checkmark$ & $\checkmark$ & success \\
\hline 47 & $\checkmark$ & $\checkmark$ & $\checkmark$ & $\checkmark$ & success \\
\hline
\end{tabular}




\begin{tabular}{|c|c|c|c|c|c|}
\hline 48 & $\checkmark$ & $\checkmark$ & $\checkmark$ & $\checkmark$ & success \\
\hline 49 & $\checkmark$ & $\checkmark$ & $\checkmark$ & $\checkmark$ & success \\
\hline 50 & $\checkmark$ & $\checkmark$ & $\checkmark$ & $\checkmark$ & success \\
\hline 51 & $\checkmark$ & $\checkmark$ & $\checkmark$ & $\checkmark$ & success \\
\hline 52 & $\checkmark$ & $\checkmark$ & $\checkmark$ & $\checkmark$ & success \\
\hline 53 & $\checkmark$ & $\checkmark$ & $\checkmark$ & $\checkmark$ & success \\
\hline 54 & $\checkmark$ & $\checkmark$ & $\checkmark$ & $\checkmark$ & success \\
\hline 55 & $\checkmark$ & $\checkmark$ & $\checkmark$ & $\checkmark$ & success \\
\hline 56 & $\checkmark$ & $\checkmark$ & $\checkmark$ & $\checkmark$ & success \\
\hline 57 & $\checkmark$ & $\checkmark$ & $\checkmark$ & $\checkmark$ & success \\
\hline 58 & $\checkmark$ & $\checkmark$ & $\checkmark$ & $\checkmark$ & success \\
\hline 59 & $\checkmark$ & $\checkmark$ & $\checkmark$ & $\checkmark$ & success \\
\hline 60 & $\checkmark$ & $\checkmark$ & $\checkmark$ & $\checkmark$ & success \\
\hline 61 & $\checkmark$ & $\checkmark$ & $\checkmark$ & $\checkmark$ & success \\
\hline 62 & $\begin{array}{ll}\checkmark & X\end{array}$ & $\checkmark$ & $\checkmark$ & $\checkmark$ & $\begin{array}{cc}\text { X: } & \text { unsuccess } \\
\checkmark & \text { success }\end{array}$ \\
\hline 63 & $\begin{array}{ll}\checkmark & X\end{array}$ & $\checkmark$ & $\checkmark$ & $\checkmark$ & $\begin{array}{cr}\text { X: } & \text { unsuccess } \\
\checkmark & \text { success } \\
\end{array}$ \\
\hline 64 & $\begin{array}{ll}\checkmark & X\end{array}$ & $\checkmark$ & $\checkmark$ & $\checkmark$ & $\begin{array}{cr}\text { X: } & \text { unsuccess } \\
\checkmark & \text { success } \\
\end{array}$ \\
\hline 65 & $\checkmark$ & $\checkmark$ & $\checkmark$ & $\checkmark$ & success \\
\hline 66 & $\checkmark$ & $\checkmark$ & $\checkmark$ & $\checkmark$ & success \\
\hline 67 & $\checkmark$ & $\checkmark$ & $\checkmark$ & $\checkmark$ & success \\
\hline 68 & $\checkmark$ & $\checkmark$ & $\checkmark$ & $\checkmark$ & success \\
\hline 69 & $\checkmark$ & $\checkmark$ & $\checkmark$ & $\checkmark$ & success \\
\hline 70 & $\checkmark$ & $\checkmark$ & $\checkmark$ & $\checkmark$ & success \\
\hline 71 & $\checkmark$ & $\checkmark$ & $\checkmark$ & $\checkmark$ & success \\
\hline 72 & $\checkmark$ & $\checkmark$ & $\checkmark$ & $\checkmark$ & success \\
\hline 73 & $\checkmark$ & $\checkmark$ & $\checkmark$ & $\checkmark$ & success \\
\hline 74 & $\checkmark$ & $\checkmark$ & $\checkmark$ & $\checkmark$ & success \\
\hline 75 & $\checkmark$ & $\checkmark$ & $\checkmark$ & $\checkmark$ & success \\
\hline 76 & $\checkmark$ & $\checkmark$ & $\checkmark$ & $\checkmark$ & success \\
\hline 77 & $\checkmark$ & $\checkmark$ & $\checkmark$ & $\checkmark$ & success \\
\hline 78 & $\checkmark$ & $\checkmark$ & $\checkmark$ & $\checkmark$ & success \\
\hline 79 & $\checkmark$ & $\checkmark$ & $\checkmark$ & $\checkmark$ & success \\
\hline 80 & $\checkmark$ & $\checkmark$ & $\checkmark$ & $\checkmark$ & success \\
\hline 81 & $\checkmark$ & $\checkmark$ & $\checkmark$ & $\checkmark$ & success \\
\hline 82 & $\checkmark$ & $\checkmark$ & $\checkmark$ & $\checkmark$ & success \\
\hline 83 & $\checkmark$ & $\checkmark$ & $\checkmark$ & $\checkmark$ & success \\
\hline 84 & $\checkmark$ & $\checkmark$ & $\checkmark$ & $\checkmark$ & success \\
\hline 85 & $\checkmark$ & $\checkmark$ & $\checkmark$ & $\checkmark$ & success \\
\hline 86 & $\checkmark$ & $\checkmark$ & $\checkmark$ & $\checkmark$ & success \\
\hline 87 & $\checkmark$ & $\checkmark$ & $\checkmark$ & $\checkmark$ & success \\
\hline 88 & $\checkmark$ & $\checkmark$ & $\checkmark$ & $\checkmark$ & success \\
\hline 89 & $\checkmark$ & $\checkmark$ & $\checkmark$ & $\checkmark$ & success \\
\hline 90 & $\checkmark$ & $\checkmark$ & $\checkmark$ & $\checkmark$ & success \\
\hline
\end{tabular}

The sample was varied into four samples, namely bulk palm oil, twice filtering palm oil, pork cooking oil without filtering and pork oil twice filtering. Each sample was tested by taking 90 experimental data, the results entered into the test table.

In this experiment, it was obtained $96.67 \%$ accuracy, because there were three sample data, namely bulk cooking oil samples detected in pig cooking oil samples without filtering. The national accuracy standard is $95 \%$, while the international accuracy standard is $96 \%$, then
96.67\% accuracy in this trial meets international standards, which means that this experiment can already be said to be a reference and can be published.

\section{CONCLUSIONS}

The resulst of this experiment shows that light intensity can distinguish the type of palm oil and pork oil with was obtained $96.67 \%$ accuracy. 


\section{REFERENCES}

Alonso M, Finn. 1992. Dasar-Dasar Fisika Universitas. Erlangga, Jakarta.

Bambang MA. 2015. Rancang Bangun Sistem Deteksi Minyak Goreng yang Telah Dipakai Menggoreng Daging Babi
Menggunakan LED dan Photodioda. [Thesis]. UIN Sunan Kalijaga, Yogyakarta. [Indonesian]

Qardhawi, Syekh Muhammad Y. 2000. Halal dan Haram dalam Pandangan Islam. Rabbaani Press, Jakarta. 
THIS PAGE INTENTIONALLY LEFT BLANK 